\title{
Ultrasonographic findings in primary umbilical endometriosis*
}

\author{
Giovanni Genovese ${ }^{1}$ \\ Stefano Veraldi ${ }^{1}$
}

\author{
Emanuela Passoni ${ }^{1}$ \\ Gianluca Nazzaro ${ }^{1}$
}

DOI: http:/ / dx.doi.org/10.1590/abd1806-4841.20187076

\begin{abstract}
Primary cutaneous endometriosis is a rare condition. It appears without a prior history of surgical procedure and the umbilicus is the most frequently involved area. Primary umbilical endometriosis, or Villar's nodule, usually presents as a painful nodule. Its differential diagnosis may be challenging. Although histopathological assessment represents the gold standard for diagnosis, cutaneous ultrasonography may be useful in guiding the surgical treatment. Ultrasonographic features of cutaneous endometriosis have not yet been fully explored in the literature. Hence, we report peculiar ultrasonographic findings of primary umbilical endometriosis
\end{abstract}

Keywords: Dermatology; Endometriosis; Ultrasonography

Endometriosis is defined as the presence of endometrial glands and stroma outside the uterine cavity. It is a benign disorder that occurs in $8-15 \%$ of women of reproductive age, representing a frequent cause of infertility. Common locations include the Fallopian tubes, followed by ovaries, uterosacral ligaments, rectovaginal septum, and pelvic peritoneum. Extrapelvic endometriosis may affect the skin in less than $5.5 \%$ of the cases. ${ }^{1}$

Contrasting ultrasonographic aspects of abdominal wall endometriosis are described in the literatureboth as homogeneously and heterogeneously hypoechoic, vascularized and non-vascularized nodules. ${ }^{2,3}$

Primary cutaneous endometriosis (PCE) - in patients without a previous history of abdominal or pelvic surgical procedures - is a rare condition, accounting for less than $30 \%$ of cases. The umbilicus is the most frequently involved region. Villar first described primary umbilical endometriosis (PUE) in 1886. It usually presents as a single red, brownish or flesh-colored nodule. Common symptoms include pain, bleeding or swelling of the lesion during periods of menstruation. However, some cases may be asymptomatic. Differential diagnoses include amelanotic melanoma, sister Mary
Joseph nodule, haemangioma, urachal remnant disease, and umbilical hernia. ${ }^{1}$

Approximately $15 \%$ of patients with PUE have coexistent pelvic endometriosis. Therefore, the diagnosis of the umbilical skin lesions may facilitate the identification and management of underlying pelvic endometriosis. First-line treatment is usually surgical excision. ${ }^{4}$

We present the case of a 42-year-old woman who was admitted to our department with a 1-month history of a painful umbilical nodule (Figure 1A). The patient had a 4-year history of pelvic endometriosis, associated with dysmenorrhea and infertility. She had undergone three ovarian stimulation procedures with gonadotropin-releasing hormone antagonists and menotropin, followed by the administration of human chorionic gonadotropin. All in vitro fertilization attempts failed. There was no history of previous open abdominal surgery or laparoscopy.

Ultrasonographic examination - carried out with a multifrequency (15.0-18.0-MHz) linear array transducer (ARIETTA V70, Hitachi Medical Systems ${ }^{\circledR}$, Zug, Switzerland) - showed an umbilical roundish nodule in the dermoepidermic layer and hypodermis,

Received on 28.02.2017.

Approved by the Advisory Board and accepted for publication on 04.07.2017.

* Work performed at the Department of Medical and Surgical Pathophysiology and Transplantation, Università degli Studi di Milano, Operative Unit of Dermatology, Fondazione Istituto di Ricovero e Cura a Carattere Scientifico(IRCCS) Ca' Granda Ospedale Maggiore Policlinico - Milan, Italy.

Financial support: None

Conflict of interest: None.

1 Department of Medical and Surgical Pathophysiology and Transplantation, Università degli Studi di Milano, Operative Unit of Dermatology, Fondazione Istituto di Ricovero e Cura a Carattere Scientifico (IRCCS) Ca' Granda Ospedale Maggiore Policlinico - Milan, Italy.

MAILING ADDRESS:

Giovanni Genovese

E-mail: giov.genov@gmail.com

C2018 by Anais Brasileiros de Dermatologia 

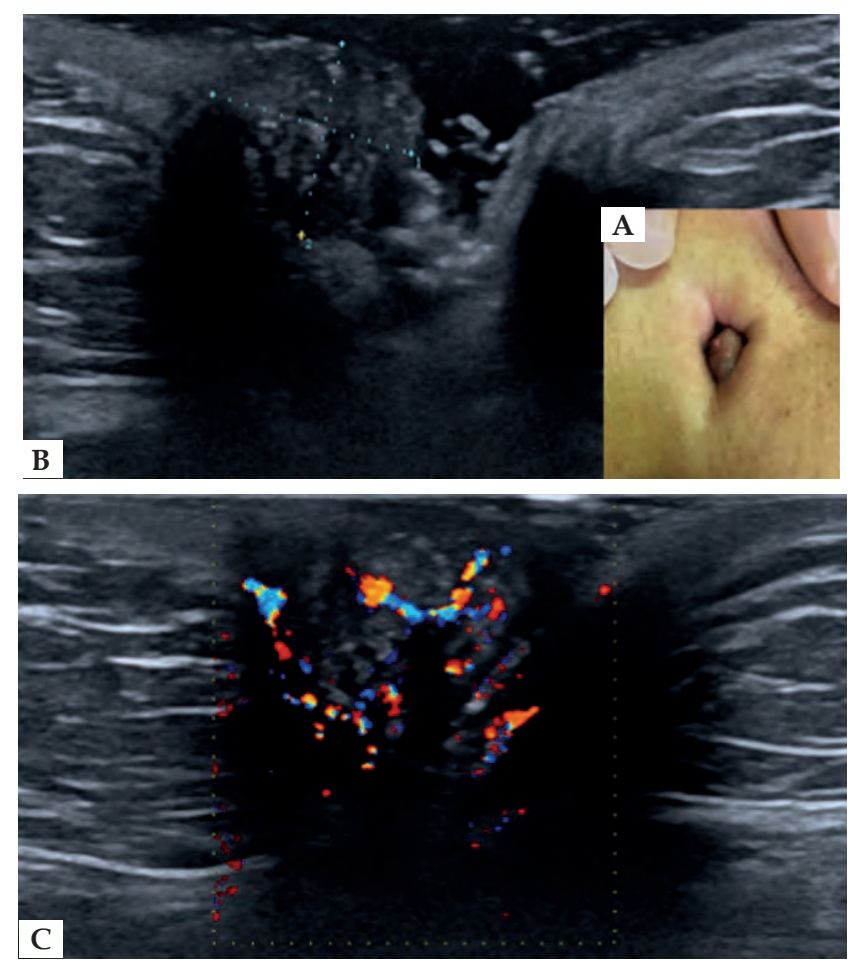

Figure 1: A - Clinical image of cutaneous endometriosis, appearing as an umbilical brownish nodule, associated with a slightly erythematous halo surrounding the navel region; B - Transverse sonographic image of the abdominal wall showing a roundish isoechoic nodule $(9.5 \times 8.5 \mathrm{~mm})$ enclosed in the subcutaneous fatty tissue, with multiple hyperechoic foci; $\mathrm{C}$ - Power Doppler examination revealed abundant blood vessels (more than seven vascular spots) within the nodule

with a non-homogeneous echotexture. The region was isoechoic relative to adjacent tissues, with multiple hyperechoic foci and abundant vessels on color Doppler examination (Figures 1B and 1C). The nodule was excised and histopathological analysis confirmed the diagnosis of PUE (Figure 2).

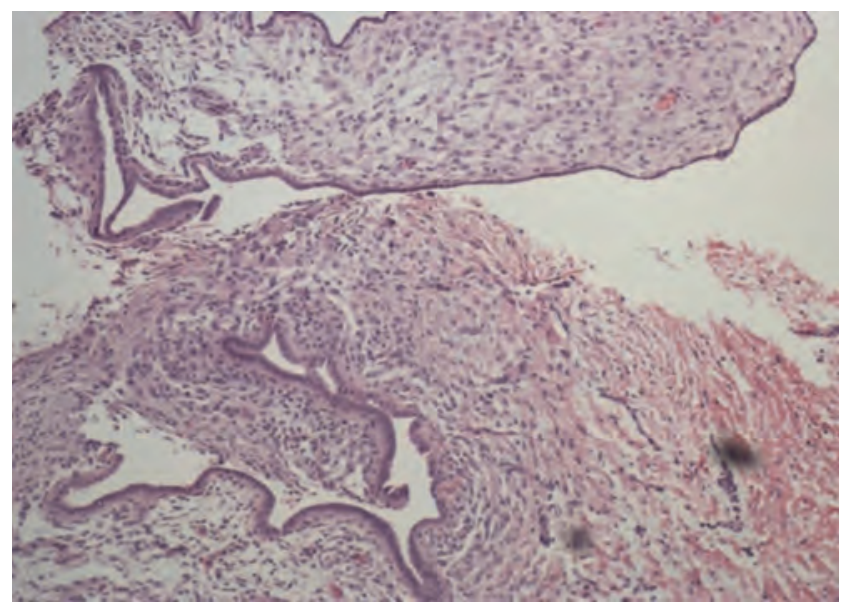

FIGURE 2: Histopathological examination showing dermal glandular proliferation of endometrial-type glands (Hematoxylin \& eosin, $\mathrm{X} 40)$.

Hence, ultrasonography can guide the surgical treatment of PUE, providing information about the nodule size and its adherence to surrounding tissues.

However, a few cases of PUE have been reported in the English literature, and only three of them were studied by means of ultrasonography. ${ }^{2,5}$ A hypoechoic pattern was found in all the above-mentioned cases. Moreover, scarce vascularization (corresponding to only one to three arterial or venous vascular spots) was found in the two cases evaluated by color Doppler. ${ }^{2}$

In conclusion, this case may be of interest because (i) PUE has never been reported in a woman undergoing ovarian stimulation and (ii) because we observed an ultrasonographic echotexture of PUE contrasting with the findings described by other authors. However, further investigations are necessary to better define PUE ultrasonographic pattern and clarify the association between PUE and ovarian stimulation. $\square$

\section{REFERENCES}

1. Nazzaro G, Coggi A, Gianotti R. A painful umbilical nodule in a 24-year-old woman. Umbilical endometriosis (Villar nodule). Int J Dermatol. 2014;53:25-6.

2. Savelli L, Manuzzi L, Di Donato N, Salfi N, Trivella G, Ceccaroni M, et al. Endometriosis of the abdominal wall: ultrasonographic and Doppler characteristics. Ultrasound Obstet Gynecol. 2012;39:336-40.

3. Hensen JH, Van Breda Vriesman AC, Puylaert JB. Abdominal wall endometriosis: clinical presentation and imaging features with emphasis on sonography. AJR Am J Roentgenol. 2006;186:616-20.
4. Moio M, Nele G. Umbilical skin endometriosis: treatment, reconstruction, and differential diagnosis. Dermatol Surg. 2017;43:449-452.

5. Theunissen $\mathrm{Cl}$, IJpma FF. Primary umbilical endometriosis: a cause of a painful umbilical nodule. J Surg Case Rep 2015;3:1-3.

How to cite this article: Genovese G, Passoni E, Veraldi S, Nazzaro G. Ultrasonographic findings in primary umbilical endometriosis. An Bras Dermatol. 2018;93(2):297-8. 